\title{
Preoperative Visualization of Cranial Nerves in Skull Base Tumor Surgery Using Diffusion Tensor Imaging Technology
}

\author{
Jun MA¹, Shaobo SU ${ }^{1}$, Shuyuan YUE${ }^{1}$, Yan $\mathrm{ZHAO}^{1}$, Yonggang $\mathrm{LI}^{1}$, Xiaochen $\mathrm{CHEN}^{1}$, Hui MA² \\ ${ }^{1}$ General Hospital of Tianjin Medical University, Division of Neurosurgery, Tianjin, China \\ ${ }^{2}$ General Hospital of Tianjin Medical University, Division of Radiology, Tianjin, China
}

\section{ABSTRACT}

AIM: To visualize cranial nerves (CNs) using diffusion tensor imaging (DTI) with special parameters. This study also involved the evaluation of preoperative estimates and intraoperative confirmation of the relationship between nerves and tumor by verifying the accuracy of visualization.

MATERIAL and METHODS: 3T magnetic resonance imaging scans including 3D-FSPGR, FIESTA, and DTI were used to collect information from 18 patients with skull base tumor. DTI data were integrated into the 3D slicer for fiber tracking and overlapped anatomic images to determine course of nerves. 3D reconstruction of tumors was achieved to perform neighboring, encasing, and invading relationship between lesion and nerves.

RESULTS: Optic pathway including the optic chiasm could be traced in cases of tuberculum sellae meningioma and hypophysoma (pituitary tumor). The oculomotor nerve, from the interpeduncular fossa out of the brain stem to supraorbital fissure, was clearly visible in parasellar meningioma cases. Meanwhile, cisternal parts of trigeminal nerve and abducens nerve, facial nerve were also imaged well in vestibular schwannomas and petroclival meningioma cases. The 3D-spatial relationship between CNs and skull base tumor estimated preoperatively by tumor modeling and tractography corresponded to the results determined during surgery.

CONCLUSION: Supported by DTI and 3D slicer, preoperative 3D reconstruction of most CNs related to skull base tumor is feasible in pathological circumstances. We consider DTI Technology to be a useful tool for predicting the course and location of most CNs, and syntopy between them and skull base tumor.

KEYWORDS: Cranial nerve, Diffusion tensor imaging, Tractography, Skull base tumor

\section{INTRODUCTION}

A dvances in neuroimaging, intraoperative monitoring, and microsurgical technique have shifted the current focus of skull base surgery from preserving life to preserving cranial nerve function. Preoperative planning using 3-dimensional (3D) visualization of image fusion has become an important development trend in skull base surgery. However, exact visualization and identification of cranial nerves (CNs) is still a challenging task with these imaging techniques due to lack of practical technology support, especially during pathological conditions. Thanks to the rapid development of diffusion tensor imaging (DTI) technology (17), it is possible now to identify the major brain white matter fibers. However, it is still difficult to achieve imaging of the small fiber bundles such as CNs. A number of recent researches have attempted to identify CNs using high-resolution or high-density DTI in subjects with or without skull base lesions $(7,20)$. The present study aimed to identify CNs in patients with skull base tumor using DTI technology with special parameters and analyze the technological parameters used. It also involved the evaluation of preoperative estimates and intraoperative confirmation of the relationship between nerves and tumor by verifying the accuracy of visualization. 


\section{MATERIAL and METHODS}

\section{Patient Demographics}

This prospective study was conducted by Department of Neurosurgery, Tianjin Medical University General Hospital. 18 subjects were enrolled in this study from October 2013 to June 2014. The mean age of the subjects was 39.5 years (range $25-60$ years), and the male-to-female ratio was $1: 1.125$. The clinical examinations of healthy subjects were normal with no structural abnormalities in magnetic resonance imaging (MRI) scan. The conditions of 18 patients were diagnosed with hypophysoma (pituitary tumor), meningioma and vestibular schwannoma in sellar, parasellar, cerebellopontine angle (CPA), and petroclival regions without any prior microsurgical or radiosurgical treatments. The study protocol was reviewed and approved by the Institutional Review Board of Tianjin Medical University General Hospital.

\section{Data Acquisition}

A Single 3T General Electric MRI scanner was used to acquire all information including the 3D-fast spoiled gradient-echo dual echo (3D-FSPGR), fast imaging employing steadystate acquisition (FIESTA), and DTI images. FSPGR set at axial view with a slice thickness of $1 \mathrm{~mm}$ was used in the $3 \mathrm{D}$ reconstruction of anatomic images. FIESTA images were collected in axial view with a $0.8 \mathrm{~mm}$ slice thickness. DTI were acquired with an 8-channel head coil using 32 diffusion sensing directions with echo planar/spin sequence (b value $1000 \mathrm{sec} /$ $\mathrm{mm}^{2}$, field of view $240 \times 240 \mathrm{~mm}$, acquired matrix $128 \times 128$ $\mathrm{mm}$, reconstructed matrix $256 \times 256 \mathrm{~mm}$, acquired voxel size $1.6 \times 1.6 \mathrm{~mm}$, slice thickness $2 \mathrm{~mm}$ ). Anatomical images and DTI digital imaging and communications in medicine (DICOM) were loaded in the 3D Slicer software as original data for tractography generation and image integration.

\section{Imaging Processing}

The DICOM files of 3D-FSPGR, FIESTA, and DTI were converted into nearly raw raster data (Nrrd) file by 3D slicer software. The new generated Nrrd file was reloaded in 3D slicer system. DTI data was used to generate a baseline volume and DTI tensor volume by least square method. Finally, the baseline volume was overlaid onto the anatomic images to complete linear registration in order to ensure the overlay accuracy of fiber bundles on T1 or FIESTA. The scientific principle and correctness of 3D slicer in fiber tracking followed the procedures discussed in related published studies (7).

\section{D Reconstruction of the Tumor}

$3 \mathrm{D}$ reconstruction of skull base space-occupying lesion was achieved via $3 d$ slicer module. It generally based on FSPGR series axial images, using Level tracing Effect and Paint Effect function of $3 d$ slicer's edit module, until completely cover all axial planes of the tumor image. After successful reconstruction, we set tumor to the transparent model, in order to observe the adjacent $\mathrm{CN}$ which associated with tumor in encasing and invading relations.

\section{Tractography Generation}

In the diffusion menu of software, fiducial or lab map seeding was chosen to set appropriate region of interest (ROI) of CNs. $\mathrm{ROI}$ was positioned in the 2-dimensional (2D) anatomical level, and T1 or FIESTA fused DTI was obtained. Osseous positioning marks such as internal auditory, optic, and Dorello canals were the reasonable placement of ROls. FIESTA images involved more skull base planes, and $\mathrm{CN}$ fibers could be clearly revealed on the axial or sagittal or coronal view in some subjects with huge space-occupying lesions. However, the ROls of some patients with space-occupying lesions could not be identified by the aforementioned method. Hence, fractional anisotropy (FA) and fibers' shortest length in seeding menu were reduced to appropriately expand the expected range of $\mathrm{ROI}$ estimated by anatomical knowledge. After the preliminary shifting of fiber tracking, the most concentrated and complete reconstructed bundle was recognized as the real $\mathrm{CN}$ fibers, which indicated that the ROI could be reset precisely in the $2 \mathrm{D}$ anatomical level based on its trajectory. Tracts were seeded by streamline tractography with an initial seed spacing of 0.5 , FA value of 0.18 , tract curvature of 0.7 , and integration step length of 0.5 . Path length was set between 20 and 800 to eliminate any unnecessary interference. FA values ranged from 0 to 1 could provide a reasonable outcome. Tractography seeding parameters could be individualized for each subject in order to minimize the interference of white matter fiber. Expect for obvious errors, the most fibers generated were not modified or pruned to ensure the least subjective disturbance.

\section{RESULTS}

Optic pathway including the optic chiasm could be traced in 2 cases of tuberculum sellae meningioma and 3 cases of hypophysoma (pituitary tumor) $(5 / 5,100 \%)$ Oculomotor nerve, from the interpeduncular fossa out of the brain stem to supraorbital fissure, was clearly visible in 2 parasellar meningioma cases. Meanwhile, trigeminal nerve allowed visualization of distal trigeminal branches and cisternal segment, and cisternal parts of abducens nerve were also imaged well in 8 vestibular schwannomas and 3 petroclival meningioma cases $(11 / 11,100 \%)$ In $90.9 \%$ of 11 posterior cranial fossa cases above, the reconstructed fibers of facial nerve were clearly visible from proximal brain stem segment to distal end extending to internal auditory canal (IAC). One exception was a case in which vestibular schwannomas was too large to enable preoperative reconstruction of facial nerve. In that case, facial nerve was exposed in anterior of tumor after partial resection during operation, while the cisternal segment presented an applanate membrane-shaped structure after complete resection. The 3D-spatial relationship between above CNs and skull base tumor estimated preoperatively by tumor modeling and tractography corresponded to the results determined during surgery (Table I). Preoperative reconstruction of other $\mathrm{CNs}$ was rarely achieved well in these cases. Each patient received surgical treatment for complete resection. Except for one patient, preoperative reconstruction of CNs was confirmed by intraoperative view of microscope and neural electrophysiological monitoring for all other patients. For that particular patient, parasellar meningioma had not yet invaded into cavernous sinus, and thus oculomotor nerve was not visible in the surgery field. 


\section{Illustrative Cases}

\section{Case 1}

A 57-year-old patient with tuberculum sellae meningioma (Figure 1A-D) is presented. The main symptoms were headache (half month), dizziness (half month), and hypopsoa (3 years). Moreover, his uncorrected visual acuity was 0.1 in the left eye and 0.15 in the right eye. Left temporal hemianopsia was found though physical examination. The following two ROls were used for tracking: orbital section and posterior optic tract. The tracking results showed that left optic nerve had come out of the optic canal and ran along the lateral surface. The posterior part was encased in tumor, while optic chiasm was located on the posterosuperior surface of tumor.
$3 \mathrm{D}$ reconstruction of the displaced and compressed right optic nerve was on the medial surface of tumor. After a partial resection of tumor through interhemispheric approach, the left optic nerve and optic chiasm were visible in the locations estimated preoperatively.

\section{Case 2}

A 29-year-old patient with parasellar meningioma (Figure $2 \mathrm{~A}-\mathrm{C})$ is presented. He suffered from persistent headache (over two years) locating left frontal-temporal area. He also presented with diplopia when looked left. Cisternal segment and superior region of petrous bone were chosen as ROls based on the FIESTA images. The reconstruction showed that nerve fiber was pushed to the lateral side of tumor, and the

Table I: Summary of the Case Results

\begin{tabular}{|c|c|c|c|c|c|c|c|}
\hline Patient & $\begin{array}{c}\text { Tumor } \\
\text { pathology }\end{array}$ & $\begin{array}{l}\text { Tumor diameter } \\
(\mathbf{c m})\end{array}$ & Tumor location & Clinical symptoms & $\begin{array}{c}\text { Related } \\
\text { nerves }\end{array}$ & $\begin{array}{l}\text { Successful } \\
\text { reconstruction }\end{array}$ & $\begin{array}{l}\text { Verified } \\
\text { intraop }\end{array}$ \\
\hline 1 & meningioma & 3.5 & $\begin{array}{l}\text { tuberculum } \\
\text { sellae }\end{array}$ & headache/dizziness & CNII & yes & yes \\
\hline 2 & meningioma & 3.2 & $\begin{array}{l}\text { tuberculum } \\
\text { sellae }\end{array}$ & - & CNII & yes & yes \\
\hline 3 & hypophysoma & 3.2 & sellar region & visual field defect & CNII & yes & yes \\
\hline 4 & hypophysoma & 3 & sellar region & visual field defect & CNII & yes & yes \\
\hline 5 & hypophysoma & 3.5 & sellar region & visual field defect & CNII & yes & yes \\
\hline 6 & meningioma & 4.5 & parasellar & diplopia & CNII-III & yes & no \\
\hline 7 & $\begin{array}{c}\text { vestibular } \\
\text { schwannoma }\end{array}$ & 3.1 & CPA & hearing impairment & CNV-VIII & yes & yes \\
\hline 6 & VS & 3.5 & CPA & $\begin{array}{c}\text { hearing impairment/ } \\
\text { tinnitus }\end{array}$ & CNV-VIII & yes & yes \\
\hline 9 & VS & 3.4 & CPA & hearing impairment & CNV-VIII & yes & yes \\
\hline 10 & VS & 4.6 & CPA & $\begin{array}{l}\text { hearing impairment/ } \\
\text { tinnitus }\end{array}$ & CNV-VIII & yes & yes \\
\hline 11 & VS & 4.2 & CPA & hearing impairment & CNV-VIII & yes & yes \\
\hline 12 & VS & 3.8 & CPA & hearing impairment & CNV-VIII & yes & yes \\
\hline 13 & VS & 4.0 & CPA & hearing impairment & CNV-VIII & yes & yes \\
\hline 14 & VS & 4.5 & CPA & $\begin{array}{l}\text { hearing impairment/ } \\
\text { facial numbness }\end{array}$ & CNV-VIII & no & - \\
\hline 15 & VS & 4.1 & CPA & $\begin{array}{l}\text { left facial numbness, } \\
\text { walking unbalance }\end{array}$ & CNV-VIII & yes & yes \\
\hline 16 & meningioma & 4 & $\begin{array}{l}\text { petroclival } \\
\text { region }\end{array}$ & left facial numbness & CN IV-VIII & yes & yes \\
\hline 17 & meningioma & 4.3 & $\begin{array}{l}\text { petroclival } \\
\text { region }\end{array}$ & $\begin{array}{l}\text { right facial numbness, } \\
\text { hearing impairment }\end{array}$ & CN IV-VIII & yes & yes \\
\hline 18 & meningioma & 4.5 & $\begin{array}{l}\text { petroclival } \\
\text { region }\end{array}$ & $\begin{array}{l}\text { left facial numbness, } \\
\text { walking unbalance }\end{array}$ & CN IV-VIII & yes & yes \\
\hline
\end{tabular}

VS: Vestibular schwannoma, CPA: Cerebellopontine angle area. 
middle part of the fiber was invaded by surrounding edema. The fiber appeared more slender morphological shape than that in healthy subjects, which could be attributed to the clinical conditions of patients such as diplopia. The orbital section of optic nerve was relatively bulky without being extruded or invaded by tumor. During operation, oculomotor nerve was not visible through transtemporal approach, prompting that tumor had indirectly squeezed the oculomotor nerve without invading into cavernous sinus.

\section{Case 3}

A 47-year-old patient with large vestibular schwannomas (Figure 3A-D) is presented. He suffered from continuous tinnitus and gradual hearing loss (two years), intermittent dizziness (recently two days). Physical examination showed right hearing impairment and his left ear heard the tuning fork sound better. Two ROls were chosen to include more involved regions for seizing the slices where facial nerve fibers were located: IAC and tumor tissue in close proximity to IAC. Using an FA level of 0.14 , facial nerve was successfully reconstructed. The tufted nerve fibers traveled out of IAC with tumor, and the applanated cisternal segment coursed along the anterior middle third of the tumor surface. Facial nerve was exposed in anterior of tumor after partial resection. The positions mentioned above were verified by electrophysiological monitoring, and the morphological shape of cisternal segment was found to be in consonance with the reconstruction results.
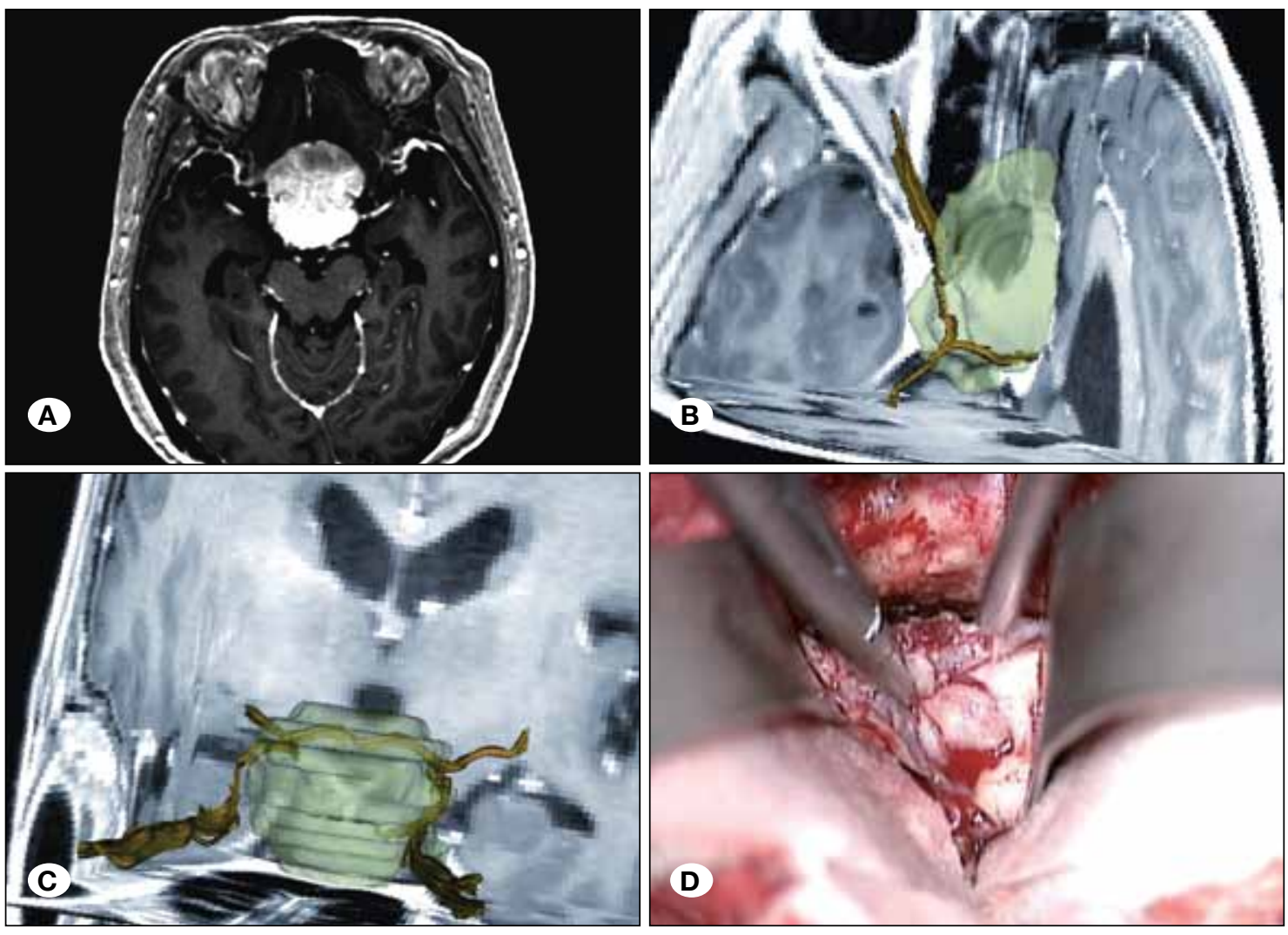

Figure 1:

(Case 1) A) MRI

image, B) Viewed from right anterior direction, the posterior part of left optic tract was encased in tumor which also pushed optic chiasm to posterior upper third of its surface. C) Posteroanterior view, D) After partial resection, left optic nerve could be observed in surgical field that encased in tumor.
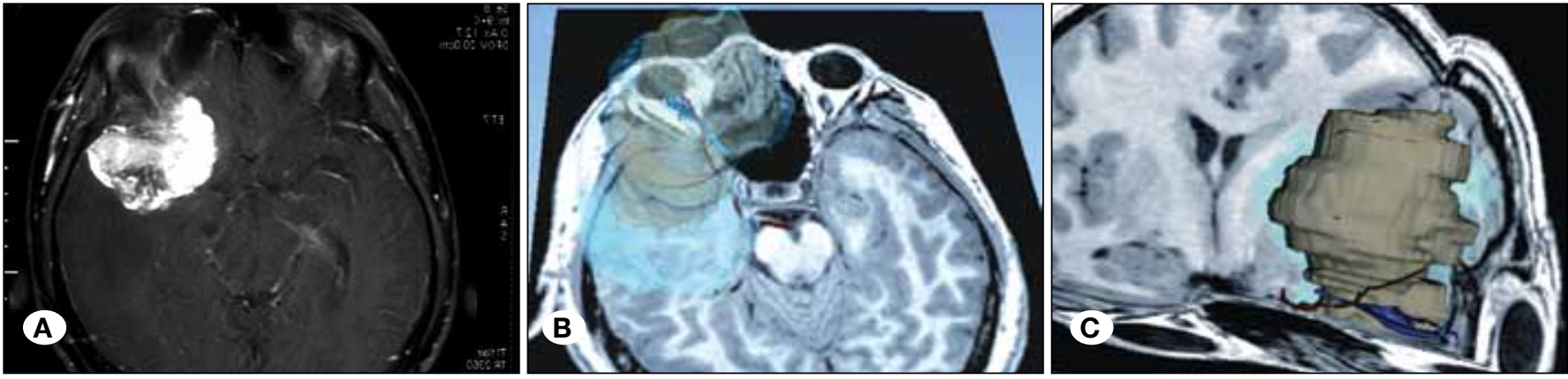

Figure 2: (Case 2) A) MRI image, B) Viewed from the right anterior direction, optic tract and oculomotor nerve were glued to internal surface of tumor and edema. C) In posteroanterior view, oculomotor nerve traveled though the lower third of the edema, and changed course of direction for being squeezed by tumor so that lead lateral angular deformity of $120^{\circ}$. 


\section{Case 4}

A 62-year-old patient with petroclival meningioma (Figure $4 A-D$ ) is presented. He admitted with gradual (over half year) walking unbalance and with left facial numbness. Upon presentation, his nasolabial groove becomes shallow on the left, with stagger and tilt to left when he was walking. Preliminary facial/vestibulocochlear nerve ROI was set within IAC. According to the trajectory of reconstruction being achieved by preliminary fiber tracking, the scope of ROI could be narrowed further. The approach for setting $\mathrm{ROI}$ of trigeminal nerve was
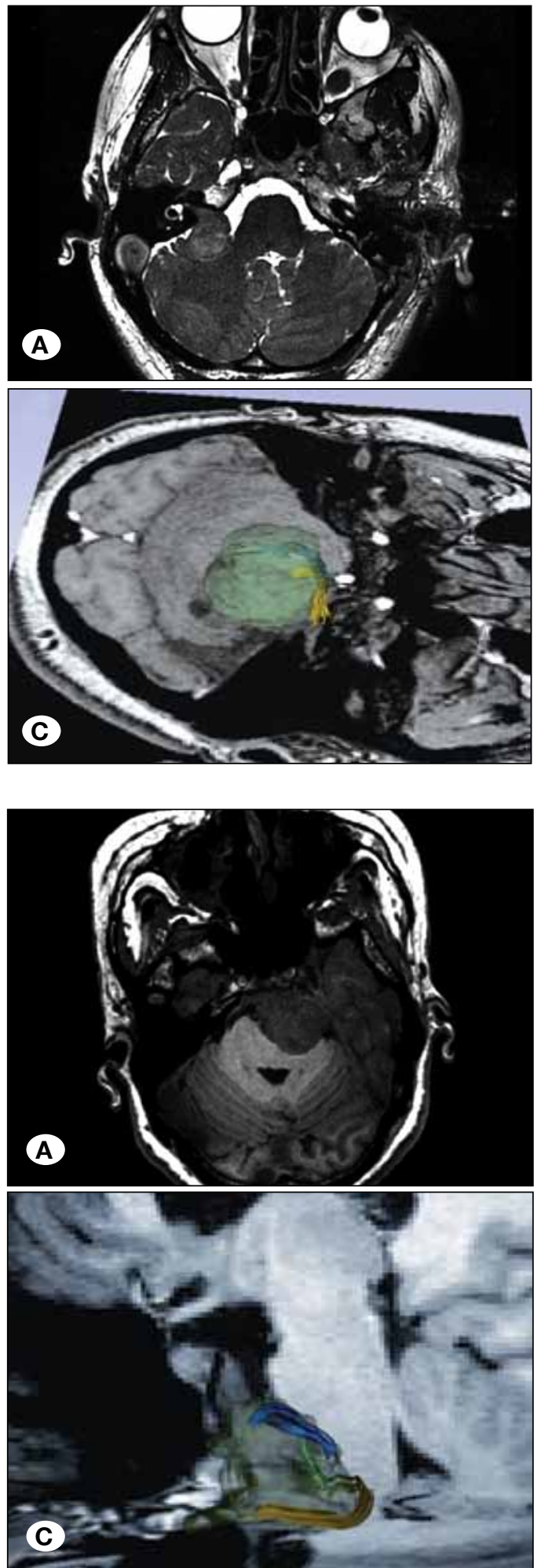
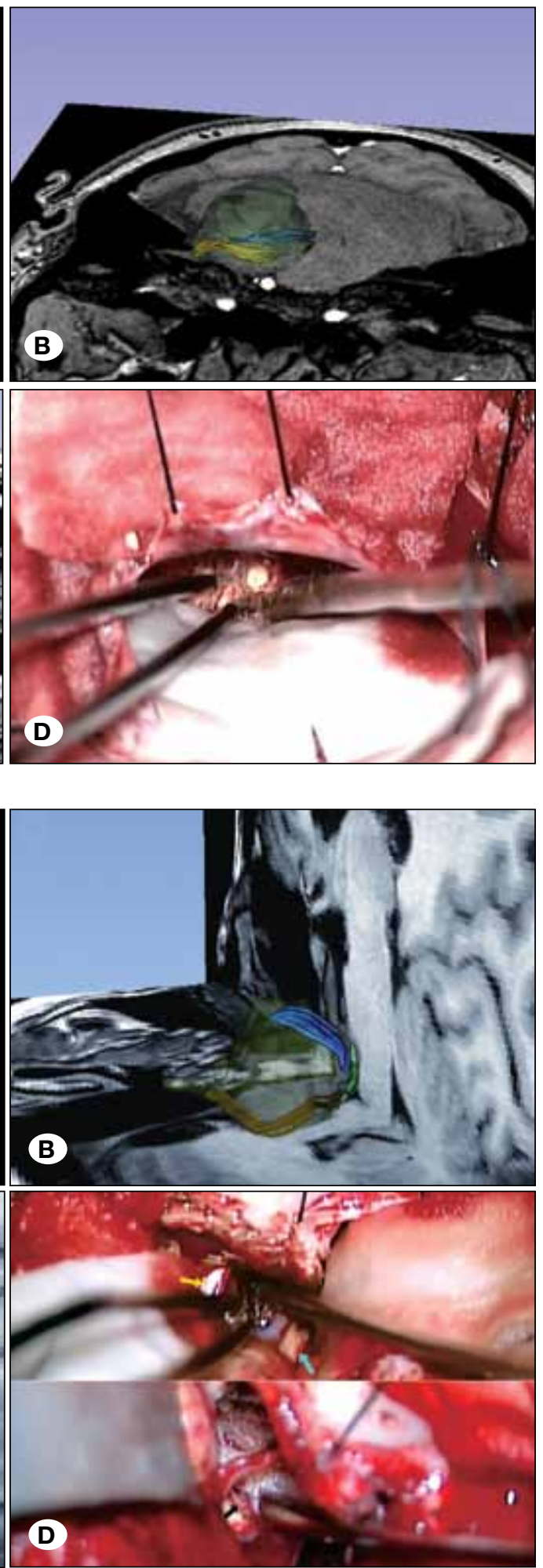

Figure 3:

(Case 3) A) MRI image, B, C) The facial nerve (yellow) was located on the anterior side of the tumor and the fibers of cisternal segment became significantly broad and thin that pressed by tumor. D) After partial resection under retrosigmoid approach, facial nerve exposed in microscopic field partly covered by tumor tissue.

Figure 4: (Case 4) A) MRI image, B, C) Image showed anterosuperior displacement of the trigeminal nerve, and facial nerve stretch cross the posterior blunt end of tumor as its course from brainstem to IAC, and abducent nerve compressed to the posteromedial surface and ascended clinging to brain stem. D) After resecting most of the tumor, the related nerves (CNV-blue arrow, CN VII-VIII-yellow arrow, CN VI-black arrow) were exposed in the surgical field. 
to fix the FIESTA slices in one step to get readily recognized on FIESTA sequence images. The ROI of abducens nerve was chosen in the posteromedial surface of tumor on the same axial slice of root entry zone (REZ). The facial/vestibulocochlear nerve stretched and crossed the posterior blunt end of tumor, bulky fibers of trigeminal nerve ran above the superior surface of the tumor, and abducens nerve was compressed to the posteromedial surface and ascended to brainstem. The location of the cranial nerves in relation to tumor determined during surgery under neural electrophysiological monitoring and direct observation corresponded to the location of fibers that were predicted by preoperative fiber tracking.

\section{- DISCUSSION}

In the recent days, considerable researches have focused on in vivo visualization of $\mathrm{CNs}$ by neuroimaging techniques. Taoka first applied a 1.5T high field MRI to track facial nerve (CN VII) by setting special parameters of DTI (24). The facial/ vestibular complex (CN VII-VIII) crossing cisternal section was successfully tracked in eight patients with vestibular schwannoma. Fujiwara, using a high-resolution 3D DTI in tiny pixel level, detected neurovascular compression in patients with trigeminal neuralgia (6). Furthermore, Roundy put forward the concept of high-density DTI under the precondition of a 3.0T high field MRI, optimized two aspects of dispersion direction with the smallest pixel precision, and implemented $3 \mathrm{D}$ reconstruction of cranial nerves; however, the study just finished with the reconstruction of facial nerve (20).

With special parameters, DTI combined with 3D slicer software to achieve tractography in our study. 3D reconstruction of most CN fibers was achieved in pathological conditions. Most of the previous reports failed in reconstruction of optic chiasm $(2,16,27)$. The present study results showed 3D body of optic pathway, especially the posterior of optic chiasm. The reconstruction of oculomotor nerve (CN III), from REZ to cavernous sinus and even superior orbital fissure, also had high fidelity. Trigeminal nerve (CN V) with the highest degree of imaging quality proved this method as a mature 3D image technology that may also be useful in predicting neurovascular compression (3). Moreover, the abducens nerve (CN VI), and the facial/vestibular nerve complex were all reconstructed well. In addition, imaging clearly show the displacement and deformation of above nerves caused by oppression and invasion of tumor.

Signal strength, ROI placement, and magnetic parameter adjustment are three key points of tractography. Macfadden had proved $3 \mathrm{~T}$ MRI could target localization more precisely than 1.5T MRI (13). Even using a 3.0T field strength MRI, it may be difficult to collect signals of some tiny nerves. Trochlear nerve could not be observed on FA chart and achieved 3D reconstruction due to its slender shape and weak DTI signals. Signal acquisition of posterior cranial nerves is barely satisfactory; and thus, search of a high-field MRI for image scanning is particularly important. Placing the ROls in an accurate area also plays a major role. For posterior cranial nerves, it is hard to locate ROIs in related anatomical layers. Setting of magnetic resonance parameters requires the assistant of a professional technician. Configuration of diffusion direction, echo time and matrix size parameters require highly specialized skills and any minor change in these parameters would affect the reconstruction results of the final fiber tracking. It is obvious that the improvement of imaging quality relies on further exploration in image parameters. Based on the experience in this study, we think that DTI image acquired from 32 directions with echo planar/spin echo sequence, and 2-mm thickness could achieve the best performance within the minimal debugging factors. Compared with other methods, it takes short time and avoided negative effect caused by long-time MRI scanning on treatment compliance, and it is also easy to operate and spread.

Application of preoperative planning system is an important embodiment of modern technology in skull base surgery. The rapid development of computer technology and radiology has enabled surgeons to conduct preoperative plan and simulate the most appropriate operation mode. Multi-model fusion imaging technology, as a new tool, has widely been used in neurosurgery recently (18). It can merge the computed tomography (CT) and MRI images together; build 3D framework of brain structure; combine with MRI angiography, CT angiography, DTI, magnetoencephalography, and other functional magnetic resonance imaging; integrate into the navigation system, and complete individualized preoperative plan (23). Functional magnetic resonance imaging confirms the orientation of cortical motor and language area. DTI can expand the scope of surgical navigation to subcortical area and accomplish preoperative assessment of pyramidal tract, radiation, and arcuate fasciculus $(11,26)$.

Surgical treatments of skull base lesions have been considered as complicated and high-risk challenges. Depending on the different location and characteristics, a space-occupying lesion could invade the bone, vessels, and CNs. Preoperative 3D visualization of the skull base structure is of vital significance for skull base surgery. The reason for high disability rate of the skull base surgery is mainly due to the damages to CNs. Detection of relative position of CNs rely on the surgeon's clinical experience and neural electrophysiological monitoring during surgery. However, personal factors are sometimes unstable, and the neural electrophysiological monitoring may be disturbed by false positive and false negative results. Without a mature 3D imaging of CNs, traditional DTI could not show intact course of CNs. Despite viewing vessel and bone images, neurosurgeons are often unable to preoperatively estimate the neighboring, encasing, and invading relations between nerves, vessels, and tumor.

In this study, we attempted to achieve 3D reconstruction of CNs related to tumor in tuberculum sellae, parasellar, CPA, and petroclival area by DTI technique. It is a tough task to design a special surgical approach that is safe enough to protect CNs. Such an approach should show the encasing and neighboring relationship between optic nerve (CN II), oculomotor nerve, trigeminal nerve, facial/vestibulocochlear nerve, and intracranial lesions. In most previous studies, optic nerve and optic chiasm in normal subjects could be tracked partly as thick fibers $(17,25)$. In the cases of optic nerve 
invaded and encased by tumor, optic chiasm and encased tract could not be reconstructed (21). 3D reconstruction of optic pathway in normal or pathological conditions was feasible in current study. In a case of tuberculum sellae tumor, optic chiasm was found to be located in the posterior upper third surface of tumor, which was encased by tumor partly. Hence, an interhemispheric approach was chosen for surgery that could help identify the optic nerve timely to avoid complication caused by operative injury. Few studies had recently reported successful 3D reconstruction of oculomotor nerve (8). In other three patients with parasellar meningioma or sellar pituitary adenoma, reconstruction could clearly reveal the deformation and displacement of $\mathrm{CN}$ II or CN III caused by compression of tumor. Intraoperatively, the position of CNs was consciously avoided to ensure accuracy of tumor resection. The current researches focus on the exploration of relationship between vestibular schwannoma and CN VII-VIII. Chen had described the preoperative basic course of CN VIII in three patients with vestibular schwannoma (4). Kyung-Sik et al. complete tracking of 11 patients' facial nerve related to large VS and proved that facial nerves course on preoperative DTT were entirely correlated with intraoperative findings in all 11 patients (12).

In 9 patients with vestibular schwannoma (with maximum diameter beyond $3 \mathrm{~cm}$ ), we successfully achieved 3D reconstruction of CN VII. Samii proved the difficulty in keeping anatomical integrity of facial nerve in a patient with large vestibular schwannoma (22). In these cases, preoperative planning position of nerve guided us to consciously avoid CN VII intraoperatively in order to ensure anatomical integrity and functional preservation. CN IV-VIII was closely related to petroclival tumor. In 3 patients with petroclival meningioma, CN V-VIII showed clear image and exact position, especially for $\mathrm{CN}$ VI whose relation with tumor evaluated by preoperative reconstruction had not been well reported. But the reconstruction of CN IV was difficult to achieve.

Application of DTI can prevent nerve damages than the traditional method based on surgeon's anatomical knowledge and intraoperative electrophysiological monitoring. Furthermore, reconstruction information of CNs could combine with vessel and bone images, overlapped anatomical MRI images, and integrated into the neuronavigation system. Neurosurgical centers can associate this technology with intraoperative MRI applications to protect the surrounding normal tissues precisely and achieve total tumor resection. Furthermore, neurosurgeons need to understand the operative technique along with imaging technology to ensure excellent surgical outcomes, and it can be improved only by proper training.

However, this technology used in this study has its limitation: DTI under 3D slicer could not reconstruct cross fibers. Fibers of CNs always mixed with neighboring bulky cross fibers in certain area, resulting in an unsatisfactory 3D reconstruction. Several other techniques have been explored to overcome this limitation, Q-ball imaging (QBI) and diffusion spectrum imaging (DSI) are the two main methods that setted with strong diffusion weightings and more diffusion directions (15). Fernandez-Miranda et al. collected diffusion spectrum imaging data of several healthy adult and 36 patients with brain lesion, and prove it has the ability to reconstruct multiple crossing of fibers and to follow the fibers through the complex crossing with a Generalized Q-Ball Imaging approach (5). While DSI is time-consuming, $\mathrm{QBI}$ could not provide useful parameter as ADC or FA for its strong diffusion weightings. Diffusional kurtosis imaging (DKI), a promising non-Gaussian diffusion-weighted imaging technique, can provide additional information derived from the tissue microstructure, as compared to conventional DTI (14). It shows excellent results in clinical applications, such as in grading gliomas, diagnosis of Parkinson disease, and evaluation of microstructural change of infarction $(9,10,19)$. Another useful function is to resolve the crossing fibers problem. Vassal et al. elaborate an approach to approximate the 3D orientation distribution function of water diffusion from DKI, and simulations of multiple fiber configurations show that could to resolve the orientations of the component fibers with the most sensitivity (26). It may distinguish fibers of gyrus rectus and orbital gyrus from olfactory nerve, and recognize different component of crossing fibers in root entry zone. Compared with QBI and DSI, DKI has its own advantage in better signal-to-noise ratio for its lower $b$ value, it may collect more slender fiber signals such as the trochlear nerve and posteriorly located nerves to achieve $3 \mathrm{D}$ reconstruction in further study. DKI also could provide significant diffusion and kurtosis parameter as Mean kurtosis (MK) to improve the sensitivity in pathological structure changes of nerve fibers compressed by tumor and edema. Atsushi et al. reconstruct fiber tracts of corticospinal tract (CST) and find increased fiber density in the CST with idiopathic normal pressure hydrocephalus, resulting in decreased MK (1). So it may also detect alterations of structural and pathological changes in $\mathrm{CNs}$ that could be helpful to diagnosis of nerve disease and assessment of nerve damage. It needs to be proved in next series of researches. Our study has another limitation, the second being the limited number of patients enrolled. Although the position of CNs adjacent to tumor was reliable under the corroboration of the intraoperative electrophysiological monitoring, a certain number of patients with intracranial space-occupying lesions in different location and different size were still needed to validate the accuracy of $\mathrm{CN}$ reconstruction. Further studies with relevant statistical analysis are recommended in order to accurately guide clinical surgeries.

\section{- CONCLUSION}

$3 \mathrm{D}$ reconstruction of $\mathrm{CN}$ fibers is feasible under the integrated application of $3 \mathrm{~T}$ high-field MRI and 3D slicer software. Most CNs can be mainly reconstructed, and their $3 \mathrm{D}$ models can be overlapped to the corresponding anatomical images. A reasonable combination of $2 \mathrm{D}$ and $3 \mathrm{D}$ models helps us to intuitively observe the course, location, and syntopy between $\mathrm{CNs}$ and intracranial space-occupying lesions. Relying on the DTI outcome, multi-image-merged navigation for preoperative planning can be united to completely restore the relationship among tumor, CNs, blood vessels, and bones. All these could eventually improve the safety factor of the skull base surgery by significantly reducing postoperative morbidity. Further application and development of this technology would greatly expand functional neurosurgery. 


\section{REFERENCES}

1. Atsushi $\mathrm{N}$, Issei $\mathrm{F}$, Masaaki $\mathrm{H}$, Yoshitaka M, Hattori T, Masakazu $M$, Shigeki A: Microstructural changes of the corticospinal tract in idiopathic normal pressure hydrocephalus: A comparison of diffusion tensor and diffusional kurtosis imaging. Neuroradiology 55(8):971-976, 2013

2. Becker M, Masterson $\mathrm{K}$, Delavelle J, Viallon M, Vargas MI, Becker CD: Imaging of the optic nerve. Eur J Radiol 74(2):299-313, 2010

3. Benes L, Shiratori K, Gurschi M, Sure U, Tirakotai W, Krischek B, Bertalanffy $\mathrm{H}$ : Is preoperative high-resolution magnetic resonance imaging accurate in predicting neurovascular compression in patients with trigeminal neuralgia? A single-blind study. Neurosurg Rev 28(2):131-136, 2005

4. Chen DQ, Quan J, Guha A, Tymianski M, Mikulis D, Hodaie M: Three-dimensional in vivo modeling of vestibular schwannomas and surrounding cranial nerves with diffusion imaging tractography. Neurosurgery 68(4):1077-1083, 2011

5. Fernandez-Miranda JC, Pathak S, Engh J, Jarbo K, Verstynen T, Yeh FC, Wang Y, Mintz A, Boada F, Schneider W, Friedlander R: High-definition fiber tractography of the human brain: Neuroanatomical validation and neurosurgical applications. Neurosurgery 71(2):430-453, 2012

6. Fujiwara S, Sasaki M, Wada T, Kudo K, Hirooka R, Ishigaki D, Nishikawa Y, Ono A, Yamaguchi M, Ogasawara K: Highresolution diffusion tensor imaging for the detection of diffusion abnormalities in the trigeminal nerves of patients with trigeminal neuralgia caused by neurovascular compression. J Neuroimaging 21(2):e102-108, 2011

7. Hodaie $M$, Quan J, Chen DQ: In vivo visualization of cranial nerve pathways in humans using diffusion-based tractography. Neurosurgery 66(4):788-795; discussion 795-796, 2010

8. Hong G, Yang Z, Chu J, Li S, Zheng S, Zhou Z: Three-dimensional MRI with contrast diagnosis of diseases involving peripheral oculomotor nerve. Clin Imaging 36(6):674-679, 2012

9. Jensen JH, Falangola MF, Hu C, Tabesh A, Rapalino O, Lo C, Helpern JA: Preliminary observations of increased diffusional kurtosis in human brain following recent cerebral infarction. NMR Biomed 24:452-457, 2012

10. Koji K, Tomiyama H, Motoi Y, Kano M, Abe O, Ito K, Shimoji K, Suzuki M, Hori M, Nakanishi A, Kuwatsuru R, Sasai K, Aoki S, Hattori N: Diffusional kurtosis imaging of cingulate fibers in Parkinson disease: Comparison with conventional diffusion tensor imaging. Magn Reson Imaging 31:1501-1506, 2013

11. Kuhnt D, Bauer MH, Becker A, Merhof D, Zolal A, Richter M, Grummich P, Ganslandt O, Buchfelder M, Nimsky C: Intraoperative visualization of fiber tracking based reconstruction of language pathways in glioma surgery. Neurosurgery 70(4):911-919; discussion 919-920, 2012

12. Kyung-Sik C, Kim MS, Kwon HG, Jang SH, Kim OL: Preoperative identification of facial nerve in vestibular schwannomas surgery using diffusion tensor tractography. J Korean Neurosurg Soc 56(1):11-15, 2014

13. MacFadden D, Zhang B, Brock KK, Hodaie M, Laperriere $N$, Schwartz M, Tsao M, Stainsby J, Lockwood G, Mikulis D, Ménard C: Clinical evaluation of stereotactic target localization using 3-Tesla MRI for radiosurgery planning. Int $\mathrm{J}$ Radiat Oncol Biol Phys 76(5):1472-1479, 2010
14. Mariana L, Jens HJ, Liang X, Helpern JA: Estimation of the orientation distribution function from diffusional kurtosis imaging. Magn Reson Med 60(4):774-781, 2008

15. Masaaki H, Issei F, Yoshitaka M, Toshiaki T, Koji K, Yuriko S, Shigeki A: Visualing non-gaussian diffusion: Clinical application of q-space imaging and diffusional kurtosis imaging of the brain and spine. Magn Reson Med Sci 11(4):221-233, 2012

16. Noble JH, Dawant BM: An atlas-navigated optimal medial axis and deformable model algorithm (NOMAD) for the segmentation of the optic nerves and chiasm in MR and CT images. Medical Image Analysis 15(6):877-884, 2011

17. Nucifora PG, Verma R, Lee SK, Melhem ER: Diffusion-tensor MR imaging and tractography: Exploring brain microstructure and connectivity. Radiology 245(2):367-384, 2007

18. Oishi M, Fukuda M, Ishida G, Saito A, Hiraishi T, Fujii Y: Presurgical simulation with advanced 3-dimensional multifusion volumetric imaging in patients with skull base tumors. Neurosurgery 68 (1 Suppl Operative):188-199; discussion 199, 2011

19. Raab P, Hattingen E, Franz K, Zanela FE, Lanfermann H: Cerebral gliomas: diffusional kurtosis imaging analysis of microstructural differences. Radiology 263:876-881, 2010

20. Roundy N, Delashaw JB, Cetas JS: Preoperative identification of the facial nerve in patients with large cerebellopontine angle tumors using high-density diffusion tensor imaging. J Neurosurg 116(4):697-702, 2012

21. Salmela MB, Cauley KA, Andrews T, Gonyea JV, Tarasiewicz I, Filippi CG: Magnetic resonance diffusion tensor imaging of the optic nerves to guide treatment of pediatric suprasellar tumors. Pediatr Neurosurg 45(6):467-471, 2009

22. Samii M, Gerganov VM, Samii A: Functional outcome after complete surgical removal of giant vestibular schwannomas. J Neurosurg 112(4):860-867, 2010

23. Tanaka $\mathrm{Y}$, Nariai T, Momose T, Aoyagi M, Maehara T, Tomori T, Yoshino Y, Nagaoka T, Ishiwata K, Ishii K, Ohno K: Glioma surgery using a multimodal navigation system with integrated metabolic images. J Neurosurg 110(1):163-172, 2009

24. Taoka T, Hirabayashi H, Nakagawa H, Sakamoto M, Myochin K, Hirohashi S, Iwasaki S, Sakaki T, Kichikawa K: Displacement of the facial nerve course by vestibular schwannoma: Preoperative visualization using diffusion tensor tractography. J Magn Reson Imaging 24(5):1005-1010, 2006

25. Techavipoo U, Okai AF, Lackey J, Shi J, Dresner MA, Leist TP, Lai S: Toward a practical protocol for human optic nerve DTI with EPI geometric distortion correction. J Magn Reson Imaging 30(4):699707, 2009

26. Vassal F, Schneider F, Sontheimer A, Lemaire JJ, Nuti C: Intraoperative visualisation of language fascicles by diffusion tensor imaging-based tractography in glioma surgery. Acta Neurochir (Wien) 155(3):437-448, 2013

27. Zhang Y, Wan SH, Wu GJ, Zhang XL: Magnetic resonance diffusion tensor imaging and diffusion tensor tractography of human visual pathway. Int J Ophthalmol 5(4):452-458, 2012 